Bull. Austral. Math. Soc.

Vol. 40 (1989) [25-35]

\title{
DECAY ESTIMATES FOR SOME NONLINEAR SECOND ORDER ORDINARY DIFFERENTIAL EQUATIONS
}

\section{MitsUHiRo NAKAO}

Precise decay estimates as $t \rightarrow \infty$ are derived for a class of nonlinear second order ordinary differential equations of the form

$$
\frac{d}{d t}\left\{h\left(\frac{d x}{d t}\right)\right\}+g\left(t, \frac{d x}{d t}\right)+f(x)=0 \text { on }(0, \infty)
$$

where $h, g$ and $f$ are functions like

$$
h(u)=|u|^{\alpha} u, g(t, u)=(1+t)^{\theta}|u|^{\beta} u, f(u)=|u|^{\gamma} u
$$

with $\alpha>-1, \beta>-1$ and $\gamma>-1$.

\section{INTRODUCTION}

In this paper we shall be concerned with the decay property of solutions of the ordinary differential equations

$$
\frac{d}{d t}\left\{h\left(\frac{d x}{d t}\right)\right\}+g\left(t, \frac{d x}{d t}\right)+f(x)=0 \text { on }(0, \infty)
$$

where $h, g, f$ are continuous functions defined on $\mathbf{R}$ or $\mathbf{R}^{+} \times \mathbf{R}\left(\mathbf{R}^{+} \equiv[0, \infty)\right)$ satisfying specific conditions described below (see Section 2).

A typical example is

$$
h(u)=|u|^{\alpha} u, g(t, u)=(1+t)^{\theta}|u|^{\beta} u, f(u)=|u|^{\gamma} u
$$

for some $\alpha>-1, \beta>-1$ and $\gamma>-1$.

For the moment let us consider the case (1.2). As is easily seen, if $\alpha=\beta=\gamma=0$ and $\theta=0$ the solutions of (1.1) decay exponentially as $t \rightarrow \infty$. Moreover, if $\alpha=0$, $\beta \geqslant 0, \gamma \geqslant 0$ and $-1 \leqslant \theta \leqslant \beta+1$ we know the following result (see $[1,2,3,6]$ )

(i) If $\theta=-1$ or $\theta=\beta+1$ and $0<\beta<\gamma$, then

$$
E(t) \equiv \frac{1}{2}|\dot{x}(t)|^{2}+\frac{1}{\gamma+2}|x(t)|^{\gamma+2} \leqslant C(E(0))\{\log (2+t)\}^{-\nu}
$$

Received 7 September 1988

Copyright Clearance Centre, Inc. Serial-fee code: 0004-9729/89 \$A2.00+0.00. 
with $\nu=(\gamma+2) /(\beta \gamma+\beta+\gamma)$.

(ii) If $-1<\theta<\beta+1$ and $\beta+\gamma>0$, then

$$
E(t) \leqslant C(E(0))(1+t)^{-\nu}
$$

with

$$
\nu=(\gamma+2) \min \left(1+\theta, \frac{\beta+1-\theta}{\beta+1}\right) /(\beta \gamma+\beta+\gamma)
$$

(iii) If $\theta<1$ and $\beta=\gamma=0$, then

$$
E(t) \leqslant C(E(0)) e^{-k t^{1-|\theta|}}
$$

with some $k=k(E(0))>0$.

The object of this paper is to extend these results to a class of more general equations including the case (1.2) with $\alpha>-1, \beta>-1$, and $\gamma>-1$. As a particular case we shall show that if $-1<\theta<\beta+1$ and $\alpha>\beta>\gamma>-1$, the solutions of (1.1) decay much faster than exponentially, that is,

$$
E(t) \equiv \frac{1}{\alpha+2}|\dot{x}(t)|^{\alpha+1}+\frac{1}{\gamma+2}|x(t)|^{\gamma+2} \leqslant C(E(0)) e^{-k e^{\nu t}}
$$

with some $k=k(E(0))>0$ and a certain $\nu>0$.

An estimate like (1.3), which seems at a glance to be very curious, is already known for a semilinear wave equation with singular nonlinearities

$$
u_{t t}-u_{x x}+|u|^{\alpha} u_{t}+|u|^{\beta} u=0,0<x<1,0<t<\infty
$$

with $0>\alpha>\beta>-1$ (see [4]). Our result tells us that such rapid decay is rather common in second order nonlinear equations.

Although the class of equations we consider is somewhat artificial it is a very convenient model for understanding how the nonlinearities influence the solutions quantitatively.

\section{ASSUMPTIONS AND RESULT}

Concerning the functions $f, g$ and $h$ appearing in (1.1) we make the following assumptions

A $1 . h(\cdot)$ belongs to $C(\mathbf{R}) \cap C^{1}(\mathbf{A}-\{0\})$ and moreover satisfies 


$$
k_{0}|u|^{\alpha} \leqslant h^{\prime}(u) \leqslant k_{1}|u|^{\alpha}(u \neq 0) \text { and } h(0)=0
$$

for some $\alpha>-1$ and positive constants $k_{0}, k_{1}$.

$\mathrm{A}_{2}, g(t, u)$ is a continuous function on $\mathbf{R}^{+} \times \mathbf{R}$ and satisfies

$$
k_{0} a(t)|u|^{\beta+2} \leqslant g(t, u) u \leqslant k_{1} b(t)|u|^{\beta+2}
$$

for some $\beta>-1, k_{0}, k_{1}>0$. Here $a(t)$ and $b(t)$ are nonnegative functions on $R^{+}$ satisfying

$$
a(t)>0 \text { a.e. and }\left\{\int_{t}^{t+1} a(s)^{-p} d x\right\}^{1 / p} \leqslant d_{0}(1+t)^{-\theta}
$$

and

$$
\int_{t}^{t+1} b(s)^{\beta+2} a(s)^{-\beta-1} d s \leqslant d_{1}(1+t)^{\theta}
$$

for some $0<p<\infty$ and $d_{0}, d_{1}>0$.

$\mathrm{A}_{3} \cdot f(\cdot)$ belongs to $C(\mathbf{R})$ and satisfies

$$
k_{0}|u|^{\gamma+2} \leqslant f(u) u \leqslant k_{1}|u|^{\gamma+1}
$$

for some $\gamma>-1$ and $k_{0}, k_{1}>0$.

We could weaken a little the assumptions above, for example, we could employ, instead of (2.2),

$$
k_{0} a(t)|u|^{\beta_{0}+2} \leqslant g(t, u) u \leqslant k_{1}\left\{b_{1}(t)|u|^{\beta_{1}+2}+b_{2}(t)|u|^{\beta_{2}+2}\right\} .
$$

To make the essential features clear, however, we restrict ourselves to the typical case $A_{1}-A_{3}$.

Since $h(u)$ may have a singularity at $u=0$ we employ the following definition of solution.

Definition 1: A function $x(\cdot)$ defined on $[0, T), 0<T<\infty$, is said to be a solution of the equation (1.1) on $[0, T)$ with the initial value $\left(x_{0}, x_{1}\right) \in \mathbf{R}^{2}$ if $x(\cdot) \in$ $C^{1}([0, T)), h(\dot{x}(\cdot)) \in C^{1}([0, T))$ and equation $(1.1)$ is satisfied on $(0, T)$ together with the initial condition $x(0)=x_{0}, \dot{x}(0)=x_{1}$.

Concerning the global existence of solution we have: 
THEOREM 1. For each $\left(x_{0}, x_{1}\right) \in \mathbf{R}^{2}$ the problem (1.1) with $x(0)=x_{0}, \dot{x}(0)=x_{1}$ admits a global solution $x(\cdot)$, that is, a solution on $[0, \infty)$.

Proof: Setting $y_{1}=h(\dot{x})$ and $y_{2}=x$ the problem is equivalent to the system

$$
\left\{\begin{array}{l}
\dot{y}_{1}=-g\left(t, h^{-1}\left(y_{1}\right)\right)-f\left(y_{2}\right) \\
\dot{y}_{2}=h^{-1}\left(y_{1}\right)
\end{array}\right.
$$

with $y_{1}(0)=h\left(x_{1}\right)$ and $y_{2}(0)=x_{0}$.

Setting also

$$
V\left(y_{1}, y_{2}\right)=\int_{0}^{h^{-1} /\left(y_{1}\right)} h^{\prime}(u) u d u+\int_{0}^{y_{2}} f(u) d u
$$

we have easily

$$
V\left(y_{1}, y_{2}\right) \geqslant C\left(\left|y_{1}\right|^{\alpha+2}+\left|y_{2}\right|^{\gamma+2}\right) \text { and } \dot{V}\left(y_{1}, y_{2}\right) \leqslant 0
$$

Thus, $V$ is a Lyapunov function for the system (2.1). The result follows immediately from this fact.

Our result on the decay property of the solutions of (1.1) reads as follows.

THEOREM 2. Let $x(t)$ be a solution of $(1.1)$ on $[0, \infty)$ and set

$$
\begin{array}{r}
E(t)=\int_{0}^{\dot{x}(t)} h^{\prime}(u) u d u+\int_{0}^{x(t)} f(u) d u \\
\left(\geqslant C\left(|\dot{x}(t)|^{\alpha+2}+|x(t)|^{\gamma+2}\right)\right) .
\end{array}
$$

We set also

$$
\eta=\max \left\{-\theta, \frac{\theta}{\beta+1}\right\} \text { and } \sigma=\min \left\{\frac{\alpha+2}{\beta+2}, \frac{(\gamma+2)(\alpha+1)}{(\gamma+1)(\beta+2)}, \frac{(\gamma+2)(\beta+1)}{(\gamma+1)(\beta+2)}\right\}
$$

(I) Assume that $\theta=-1$ or $\beta+1$ and let $\sigma<1$. Then we have

$$
E(t) \leqslant C(E(0))\{\log (2+t)\}^{-\nu}
$$

with $\nu=\sigma /(1-\sigma)$.

(II) Assume that $-1<\theta<\beta+1$ and $\sigma<1$. Then

$$
E(t) \leqslant C(E(0))(1+t)^{-\nu}
$$

with $\nu=(1-\eta) \sigma /(1-\sigma)$.

(III) Assume that $-1<\theta<\beta+1$ and let $\alpha=\beta>\gamma$ or $\alpha>\beta=\gamma$. Then 


$$
E(t) \leqslant C(E(0)) e^{-k t^{1-\eta}}
$$

where $k$ is a positive constant depending on $E(0)$ and other known constants.

(IV) Assume that $-1<\theta<\beta+1$ and $\sigma>1$, that is, $\alpha>\beta>\gamma$. Then

$$
E(t) \leqslant C(E(0)) e^{-k e^{(\nu-e) t}}, \quad 0<\varepsilon<\nu
$$

with $\nu=\log \sigma(>0)$, where $k$ is a positive constant depending on $E(0)$ and $\varepsilon$. We can take $\varepsilon=0$ if $\theta=0$.

Remark. When $\theta=-1$ or $\beta+1$ and $\sigma=1$ we can show, instead of (2.11),

$$
E(T) \leqslant C(E(0))(1+t)^{-\nu}
$$

for some $\nu>0$ depending on $E(0)$.

\section{Some Lemmas}

The following lemma is essential for precise estimation of the solutions.

LEMMA 1. Let $\phi(t)$ be a nonnegative function on $\mathbf{A}^{+}=[0, \infty)$, satisfying the difference inequality

$$
\sup _{t \leqslant s \leqslant t+1} \phi(s)^{1+r} \leqslant C_{0}(1+t)^{\theta}(\phi(t)-\phi(t+1))+\delta(t)
$$

for some $C_{0}>0, \theta<1, r \geqslant 0$ and $\delta(t)$ a bounded function on $\mathrm{P}^{+}$. Then, $\phi(t)$ has the following decay property

(i) if $\theta=1, r>0$ and $\delta(t)=0\left((\log t)^{-1-1 / r}\right)$ as $t \rightarrow \infty$. then

$$
\phi(t) \leqslant C(\phi(0))\{\log (2+t)\}^{-1 / r}
$$

(ii) if $\theta<1, r>0$ and $\delta(t)=0\left(t^{-(1-\theta)(1+1 / r)}\right)$ as $t \rightarrow \infty$, then

$$
\phi(t) \leqslant C(\phi(0))(1+t)^{-(1-\theta) / r}
$$

(iii) if $\theta<1, r=0$ and $\delta(t)=0\left(e^{-t^{1-\theta}}\right)$ as $t \rightarrow \infty$, then

$$
\phi(t) \leqslant C(\phi(0)) e^{-k t^{1-\theta}}
$$

$$
\text { for some } k=k(\phi(0))>0 \text {. }
$$

For the proof of Lemma 1 see [2] or Redheffer and Walter [5]. Using Lemma 1 we can obtain 
LEMмA 2. Let $\phi(t)$ be a decreasing function on $\mathbf{R}^{+}$, satisfying

$$
\phi(t) \leqslant C_{0} \sum_{i=1}^{n}(1+t)^{\theta_{i}}(\phi(t)-\phi(t+1))^{\alpha_{i}}
$$

for some $C_{0}>0$. Then $\phi(t)$ has the following decay property

(i) if $0<\min _{1 \leqslant i \leqslant n}\left\{\alpha_{i}\right\} \equiv \sigma<1$ and $\max _{1 \leqslant i \leqslant n}\left\{\theta_{i} / \alpha_{i}\right\} \equiv \eta=1$, then

$$
\phi(t) \leqslant C(\phi(0))\{\log (2+t)\}^{-\nu}
$$

with $\nu=\sigma /(1-\sigma)$;

(ii) if $0<\sigma<1$ and $\eta<1$, then $\phi(t) \leqslant C(\phi(0))(1+t)^{-\nu}$ with $\nu=$ $(1-\eta) \sigma /(1-\sigma)$;

(iii) if $\sigma=1$ and $\eta<1$, then $\phi(t) \leqslant C(\phi(0)) \exp \left\{-k t^{1-\eta}\right\}$ for some $k=$ $k(\phi(0))>0$.

Proof: All cases can be proved similarly, and we give the proof only for case (ii). First, note that if $\alpha_{i} \leqslant \alpha_{j}$ and $\theta_{i} \leqslant \theta_{j}$ for some $i, j$ we can remove the term $(1+t)^{\theta_{j}}(\phi(t)-\phi(t+1))^{\theta_{j}}$ from the right-hand side of (3.2). Therefore, without loss of generality, we may assume

$$
\alpha_{1}>\alpha_{2}>\ldots>\alpha_{n} \text { and } \theta_{1}>\theta_{2}>\ldots>\theta_{n}
$$

Then, from (3.2) we have

$$
\min _{1 \leqslant i \leqslant n} \phi(t)^{1 / \alpha_{i}} \leqslant C_{0} n \max _{1 \leqslant i \leqslant n}(1+t)^{\theta_{i} / \alpha_{i}}(\phi(t)-\phi(t+1))
$$

and hence

$$
\begin{aligned}
\phi(t)^{1 / \alpha_{n}} & =\left\{\frac{\phi(t)}{\sup \phi(s)}\right\}^{1 / \alpha_{n}} \sup \phi(s)^{1 / \alpha_{\mathfrak{n}}} \\
& \leqslant\{\phi(t) / \phi(0)\}^{1 / \alpha_{i}} \phi(0)^{1 / \alpha_{n}}(\forall i) \\
& \leqslant C(\phi(0))(1+t)^{\eta}(\phi(t)-\phi(t+1))
\end{aligned}
$$

with $\eta=\max _{1 \leqslant i \leqslant n} \theta_{i} / \alpha_{i}$.

Thus, applying Lemma 1 (ii) to (3.3) we have the desired estimate.

LEMMA 3. Let $\phi(t)$ be a nonnegative decreasing function on $\mathbf{R}^{+}$, satisfying

$$
\phi(t) \leqslant C_{0} e^{-k_{0} t^{1-\theta}} \text { for } t \in \mathbf{R}^{+}
$$


with some $C_{0}, k_{0}>0$ and $\theta<1$, and the difference inequality

$$
\phi(t+1) \leqslant C_{1} \sum_{i=1}^{n}(1+t)^{\theta_{i}} \phi(t)^{\alpha_{i}}
$$

with $C_{1}>0$ and $\theta_{i}, \alpha_{i}$ such that

$$
\sigma \equiv \min _{1 \leqslant i \leqslant n}\left\{\alpha_{i}\right\}>1 \text { and } \eta \equiv \max _{1 \leqslant i \leqslant n}\left\{\alpha_{i} / \theta_{i}\right\}<1
$$

Then, for any $0<\varepsilon<<1$ there exist $C_{\varepsilon}=C(\varepsilon, \phi(0))$ and $k=k(\varepsilon, \phi(0))$ such that

$$
\phi(t) \leqslant C_{\varepsilon} e^{-k e^{(\nu-c) t}} \text { for } t \geqslant 0
$$

where we set $\nu=\log \sigma(>0)$. When $\eta=0$ we can take $\varepsilon=0$ in (3.5).

Proof: It follows from (3.4) that

$$
\min _{1 \leqslant i \leqslant n} \phi(t+1)^{1 / \alpha_{i}} \leqslant C_{1} n \max _{1 \leqslant i \leqslant n}(1+t)^{\theta_{i} / \alpha_{i}} \phi(t)
$$

and hence

$$
\phi(t+1)^{1 / \sigma} \leqslant C(1+t)^{\eta} \phi(t)
$$

for some $C>0$.

By the assumption on the decay of $\phi(t)$ as $t \rightarrow \infty$ we see that for any $\varepsilon>0$, there exists $T_{\varepsilon}>0$ such that

$$
C^{\sigma}(1+t)^{\eta \sigma} \phi(t)^{\sigma} \leqslant \phi(t)^{\sigma-\varepsilon} \quad \text { if } t \geqslant T_{\epsilon} .
$$

Therefore we have from (3.6) that

$$
\phi(t) \leqslant \phi(t-1)^{\sigma-\varepsilon} \leqslant \phi(t-m)^{(\sigma-e)^{m}} \quad \text { if } t-m \geqslant T_{\varepsilon}
$$

and

$$
\phi(t) \leqslant \phi\left(T_{e}\right)^{(\sigma-e)^{[t-T e]}} \quad \text { if } t \geq T_{\epsilon}
$$

where $\left[t-T_{e}\right]$ denotes the integer part of $t-T_{\varepsilon}$. Since we may assume $\phi\left(T_{\varepsilon}\right)<e^{-1}$ it follows from (3.8) that

$$
\begin{aligned}
\phi(t) & \leqslant e^{-e^{\left[t-T_{e}\right] \log (\sigma-\varepsilon)}}(0<\varepsilon<\sigma) \\
& \leqslant e^{-k e^{\nu_{e} t}} \text { if } t>T_{\varepsilon}
\end{aligned}
$$

with $\nu_{e}=\log (\sigma-\varepsilon)>0$. Changing the notation yields (3.5).

It is clear that when $\eta=0$ we can take $\varepsilon=0$ and $T_{e}=0$ in (3.7) and the estimate (3.5) holds with $\nu_{e}=\nu=\log \sigma$. 


\section{Proof of Theorem 2}

Let $x(\cdot)$ be a solution of (1.1) (in the sense of Definition 1) and let us recall that

$$
E(t)=\int_{0}^{\dot{x}(t)} h(u) u d u+\int_{0}^{x(t)} f(u) d u
$$

We note again that

$$
\begin{aligned}
& k_{0}\left\{\frac{1}{\alpha+2}|\dot{x}(t)|^{\alpha+2}+\frac{1}{\gamma+2}|x(t)|^{\gamma+2}\right\} \\
& \quad \leqslant E(t) \leqslant k_{1}\left\{\frac{1}{\alpha+2}|\dot{x}(t)|^{\alpha+2}+\frac{1}{\gamma+2}|x(t)|^{\gamma+2}\right\}
\end{aligned}
$$

$\left(k_{0}, k_{1}>0\right)$.

Multiplying the equation (1.1) by $\dot{x}(t)$ and integrating over $[t, t+1]$ we have

$$
\int_{t}^{t+1} g(s, \dot{x}(s)) \dot{x}(s) d s=E(t)-E(t+1) \equiv D(t)^{\beta+2}
$$

and by the assumption $A_{2}$

$$
k_{0} \int_{t}^{t+1} a(s)|\dot{x}(s)|^{\beta+2} d s \leqslant D(t)^{\beta+2}
$$

In what follows $C$ will denote generous positive constants, in particular $C(\phi(0))$ will denote constants depending on $\phi(0)$ continuously.

Now, with the use of the assumption on $a(\cdot)$ we have, for any $r>0$,

$$
\begin{aligned}
& \int_{t}^{t+1}|\dot{x}(s)|^{r} d s=\int_{t}^{t+1} a(s)^{-p /(p+1)} a(s)^{p /(p+1)}|\dot{x}(s)|^{r} d s \\
& \leqslant\left\{\int_{t}^{t+1} a(s)^{-p} d s\right\}^{1 /(p+1)}\left\{\int_{t}^{t+1} a(s)|\dot{x}(s)|^{r(p+1) / p} d s\right\}^{p /(p+1)} \\
& \leqslant C(1+t)^{-p \theta /(p+1)}\left\{\int_{t}^{t+1} a(s)|\dot{x}(s)|^{\beta+2} d s\right\}^{p /(p+1)} \\
& \sup _{t \leqslant s \leqslant t+1}|\dot{x}(s)|^{r-p(\beta+2) /(p+1)} \\
& \leqslant C(1+t)^{-p \theta /(p+1)} D(t)^{p(\beta+2) /(p+1)} \\
& \sup _{t \leqslant s \leqslant t+1} E(s)^{\{r(p+1)-p(\beta+2)\} /(\alpha+2)(p+1)}
\end{aligned}
$$

where we have assumed that $r(p+1) \geqslant p(\beta+2)$. 
Taking $r=p(\beta+2) /(p+1)$ in (4.4) we see that

$$
\int_{t}^{t+1}|\dot{x}(s)|^{p(\beta+2) /(p+1)} d s \leqslant C(1+t)^{-p \theta /(p+1)} D(t)^{p(\beta+2) /(p+1)}
$$

and hence there exist $t_{1} \in[t, t+1 / 4], t_{2} \in[t+3 / 4, t+1]$ such that

$$
\left|\dot{x}\left(t_{i}\right)\right| \leqslant C(1+t)^{-\theta /(\beta+2)} D(t), i=1,2 .
$$

Next, multiplying the equation (1.1) by $x(t)$ and integrating over $\left[t_{1}, t_{2}\right]$ we have

$$
\begin{aligned}
\int_{t_{1}}^{t_{2}} f(x(s)) x(s) d s= & -\int_{t_{1}}^{t_{2}} h(\dot{x}(s)) \dot{x}(s) d s-h\left(\dot{x}\left(t_{2}\right)\right) x\left(t_{2}\right) \\
& +h\left(\dot{x}\left(t_{1}\right)\right) x\left(t_{1}\right)-\int_{t_{1}}^{t_{2}} g(s, \dot{x}(s)) x(s) d s \\
\leqslant & C\left\{\int_{t_{1}}^{t_{2}}|\dot{x}(s)|^{\alpha+2} d s\right. \\
& +\sum_{i=1}^{2}\left|\dot{x}\left(t_{i}\right)\right|^{\alpha+1} \sup _{t \leqslant s \leqslant t+1}|x(s)| \\
& \left.+\int_{t_{1}}^{t_{2}} b(s)|\dot{x}(s)|^{\beta+1}|x(s)| d s\right\} .
\end{aligned}
$$

Each term of the righthand side of (4.6) is treated as follows.

First, without loss of generality, we may assume $p$ is sufficiently small and we can take $r=\alpha+2$ in (4.4) to get

$$
\begin{aligned}
& \int_{t_{1}}^{t_{2}}|\dot{x}(s)|^{\alpha+2} d s \leqslant C(1+t)^{-p \theta /(p+1)} D(t)^{p(\beta+2) /(p+1)} \\
& \times \sup _{t \leqslant s \leqslant t+1} E(s)^{1-p(\beta+2) /(\alpha+2)(p+1)} .
\end{aligned}
$$

By (4.5),

$$
\begin{aligned}
\sum_{i=1}^{2}\left|\dot{x}\left(t_{i}\right)\right|^{\alpha+1} \sup _{t \leqslant s \leqslant t+1}|x(s)| \leqslant & C(1+t)^{-\theta(\alpha+1) /(\beta+2)} D(t)^{\alpha+1} \\
& \times \sup _{t \leqslant s \leqslant t+1} E(s)^{1 /(\gamma+2)} .
\end{aligned}
$$


Finally,

$$
\begin{aligned}
& \int_{t_{1}}^{t_{2}} b(s)|\dot{x}(s)|^{\beta+1}|x(s)| d s \\
& \leqslant\left\{\int_{t_{1}}^{t_{2}} a(s)|\dot{x}(s)|^{\beta+2} d s\right\}^{(\beta+1) /(\beta+2)}\left\{\int_{t_{1}}^{t_{2}} b(s)^{\beta+2} a(s)^{-\beta-1} d s\right\}^{1 /(\beta+2)} \\
& \quad \times \sup _{t \leqslant s \leqslant t+1}|x(s)| \\
& \leqslant C D(t)^{\beta+1}(1+t)^{\theta /(\beta+2)} \sup _{t \leqslant s \leqslant t+1} E(s)^{1 /(\gamma+2)} .
\end{aligned}
$$

Thus, we have from (4.6)

$$
\begin{aligned}
E\left(t_{2}\right) \leqslant C & \int_{t_{1}}^{t_{2}}\left\{\frac{1}{\alpha+2}|\dot{x}(s)|^{\alpha+2}+\frac{1}{\gamma+2}|x(s)|^{\gamma+2}\right\} d s \\
\leqslant C & \left\{(1+t)^{-p \theta /(p+1)} D(t)^{p(\beta+2) /(p+1)} \sup _{t \leqslant s \leqslant t+1} E(s)^{1-p(\beta+2) /(\alpha+2)(p+1)}\right. \\
& +(1+t)^{-\theta(\alpha+1) /(\beta+2)} D(t)^{\alpha+1} \sup _{t \leqslant s \leqslant t+1} E(s)^{(1 / \gamma+2)} \\
& \left.+(1+t)^{\theta /(\beta+2)} D(t)^{\beta+1} \sup _{t \leqslant s \leqslant t+1} E(s)^{1 /(\gamma+2)}\right\} \equiv A(t)
\end{aligned}
$$

Furthermore, by (4.2) and (4.7),

$$
\begin{aligned}
\sup _{t \leqslant s \leqslant t+1} E(s) & \leqslant E\left(t_{2}\right)+\int_{t}^{t+1} g(s, \dot{x}(s) \dot{x}(s)) d s \\
& \leqslant A(t)+D(t)^{\beta+2}
\end{aligned}
$$

and consequently

$$
\begin{aligned}
E(t)= & \sup _{t \leqslant s \leqslant t+1} E(s) \leqslant C\left\{(1+t)^{-\theta(\alpha+2) /(\beta+2)} D(t)^{\alpha+2}\right. \\
& +(1+t)^{-\theta(\alpha+1)(\gamma+2) /(\gamma+1)(\beta+2)} D(t)^{(\alpha+1)(\gamma+2) /(\gamma+1)} \\
& +(1+t)^{(\gamma+2) \theta /(\beta+2)(\gamma+1)} D(t)^{(\beta+1)(\gamma+2) /(\gamma+1)} \\
& \left.+D(t)^{\beta+2}\right\} .
\end{aligned}
$$

From (4.7) we obtain also

$$
\begin{aligned}
E(t+1) \leqslant C & \left\{(1+t)^{-p \theta /(p+1)} E(t)^{1+p(\alpha-\beta) /(\alpha+2)(p+1)}\right. \\
& +(1+t)^{-\theta(\alpha+1) /(\beta+2)} E(t)^{(\alpha+1) /(\beta+2)+1 /(\gamma+2)} \\
& +(1+t)^{\theta /(\beta+2)} E(t)^{(\beta+1) /(\beta+2)+1 /(\gamma+2)} .
\end{aligned}
$$


Now, we can apply Lenma 2 to the inequality (4.8) to get the estimates (I)-(III) in Theorem 2. When $\sigma>1$, namely, $\alpha>\beta>\gamma$ it is first verified from (4.8) and Lemma 2 (iii) that

$$
E(t) \leqslant C(E(0)) e^{-k t}
$$

with some $k>0$ under the condition $-1<\theta<\beta+1$, and hence application of Lemma 3 to (4.9) yields the estimate (IV) in Theorem 1. The proof of Theorem 2 is now completed.

\section{REFERENCES}

[1] M. Nakao, 'On bounded, periodic and almost periodic solutions for a system of nonlinear second order ordinary differential equations', J. Math. Anal. Appl. 61 (1977), 620-632.

[2] M. Nakao, 'A difference inequality and its application to nonlinear evolution equations', $J$. Math. Soc. Japan 30 (1978), 747-762.

[3] M. Nakro, 'Asymptotic stability for some nonlinear evolution equations of second order with unbounded dissipative term', J. Differential Equations 30 (1978), 54-63.

[4] M. Nakao, 'An example of nonlinear wave equation whose solutions decay faster than exponentially', J. Math. Anal. Appl. 122 (1987), 260-264.

[6] R. Redheffer and W. Walter, 'A comparison theorem for difference inequalities', J. Differential Equations 44 (1982), 111-117.

[6] Y. Yamada, 'On the decay of solutions for some nonlinear evolution equations of second order', Nagoya Math. J. 1 (1979), 67-98.

Department of Mathematics

College of General Education Kyushu University

Fukuoka 810

Japan 